\title{
Abstraction as a limit to semiosis
}

\section{Tahir Wood}

\begin{abstract}
:
In highly evolved culture, discourse is made up of complexes of implicit and explicit intertextual relations, which form the meanings for new signifiers. Meanings for common abstract nouns are derived from the modeling of typical situations in everyday narratives. However at a further level of abstraction, models of discourses, which themselves contain abstract concepts, provide meanings for what are called "hyper-abstract" nominals. Here a certain limit is reached, and it is argued that this diachronic, onomasiological process provides a constraint on the notion of "unlimited semiosis." This constraint has both natural and ethical aspects.
\end{abstract}

\section{Introduction}

This famous triad of icon, index, and symbol cannot be understood today as a taxonomy of three "kinds" of signs, as first suggested by Peirce (1894: §3). But instead of discarding it I would prefer to understand it as indicating three aspects of a sign, and my choice of the term triad, as opposed to "trichotomy" in Eco's (1976) usage, recalls the triad of notes that together make up a basic musical chord.

In human semiosis these three aspects of the sign are invariably co-present, while in the semiotics of other species the symbolic is either absent or very much less developed. In these species the operation of the index is innately specified to a greater extent, so that

... unlike the mechanical operations of fixed, predetermined tropisms that are genetically hard-wired behavioral patterns belonging to animals and lower organisms, human drives are determinative. That is, they are endowed with a degree of freedom manipulated by the agency of the ego, an ego that operates on manifold levels of conscious and unconscious

activity. (Mills 2004: 675)

But a degree of continuity between the various animal species and the human should not be denied either, since "the human Umwelt is first of all an animal Umwelt, a species-specific objective world, but it is based on a biologically underdetermined Innenwelt or modeling system" (Deely 2004: 20). It is the symbolic 
order that in its own way fills out the space of biological under-determining mentioned by Deely. This will be explained using the zoosemiotics of René Thom.

What I would like to demonstrate is that the symbolic order that enables human understanding, is defined paradoxically by a certain property, which, the more that it is realized, the greater its potential to lead to human misunderstanding. This property is abstraction. Thus, whatever may be understood by the term "unlimited semiosis," there is one thing that it cannot mean, and that is rising abstraction without limit.

\section{The animal kingdom, the icon, and the index}

The iconic is that fundamental aspect of the sign whereby something is represented in a non-arbitrary manner. This includes such phenomena as the imprint in a plastic medium, for example, a footprint in the sand, or a reflection, such as a face in a mirror. But it is not always noted how it also includes the full range of phenomena of perception. Animal organisms have an inner representation of their environment, including a mapping of the surrounding territory. Features of the environment are imprinted, so to speak, onto the perceptual apparatus of the organism. This inner representation cannot be arbitrary if the organism is to survive; it must of necessity be iconic in nature, i.e., based on the principle of isomorphism, the core aspect of the "genesis of the image" (Thom 1983: 262).

But not every image in the environment is particularly significant to the organism, or pregnant, in Thom's terminology; only as an index does this become the case. The index includes the iconic aspect within itself, but adds the pregnancy that is absent from the iconic on its own. The index is based on associations, some learned and some apparently innate ("hardwired"), among the various objects of perception that are of interest to the organism. Such objects obtain their interest for the animal due to an association, for example the spoor that indicates the path of a pursued prey. Thom explains this association by saying that the index "is always an actant which is, or has been, in contact with its object, if it is not actually part of it" (1983: 267).

There is a considerable distance travelled, however, in the evolution of the cognitive faculty in the process of hominization, resulting in much more complex expressions of meaning, including the fact that there can be a meaningful expression freed from the identification of a unique individual or stimulus in the immediate environment, so that "human language allows the description of a distant process (in space and time) and frees the mind from the tyranny of the 'here and now' to which the animal remains subject" (Thom 1983: 275). It is in the symbolic realm that this becomes possible.

\section{The symbolic realm}

The symbolic is defined in terms of several related capacities: first, the capacity for a more contingent relationship between the signifier and that which is signified - we should not go so far as to claim, however, that such relations are 
always and entirely arbitrary; second, the capacity to retain and distribute many of these signifiers differentially across various meanings (allowing also for polysemy); third, the capacity to manipulate and aggregate them in a wide range of combinatorial forms. Sebeok (1999) accounts for the emergence of the symbolic in terms of two stages:

. . language emerged as an evolutionary adaptation over two million years ago, in the guise of a mute semiotic modeling system - briefly a tool wherewith hominids analyze their surroundings - and was thus present in Homo habilis and all successor species. Speech, the paramount linear display of language in the vocal-auditory mode, appeared as a secondary exaptation probably less than 100,000 years ago, the minimum time required to adjust a species-specific mechanism for encoding sentences with a matching mechanism for decoding and interpreting them in another brain. The fine-tuning process continues. (Sebeok 1999: 92)

The initial challenge for us is always to understand how it is that symbolic resources, including the vast resources of the world's languages, depend upon and incorporate the iconic and indexical aspects of signs while going far beyond these. But then we must come to understand how the further evolution of culture brings new complexities into the web of semiosis that surely go beyond Sebeok's seemingly modest notion of "fine-tuning."

So in hominization the iconic and indexical are somehow taken up into a new synthesis, the symbolic order. As Wildgen (1982: 20) puts it, "these aspects are ordered insofar as Index uses iconic principles and Symbol makes use of iconic and indexical devices." A sign that does not go beyond the indexical principle is relatively immediate and concrete, even though it may be learned and even arbitrary like the sound of the bell to Pavlov's dogs.

Now where Thom explains the emergence of the abstract as the merging of territorial maps into one unified conception of space, thereby bringing about the unified ego in man, we might go further and understand, more generally, how it is that powers of abstraction make concepts, and hence the entire symbolic order possible. It is not just the fact that we can have a general notion of space beyond the maps of distinct territorial spaces that is decisive for the emergence of the symbolic, but rather that we can generalize any and every sort of phenomenon into a universal category or relation. Stjernfelt has this way of putting the matter:

Higher animals may not only recognize tokens as instantiations of types, they may make use of these types to symbolize, to reason, argue, use diagrams. Probably, the special human privilege is abstraction, making it possible for us to make explicit and contemplate such types, reasonings, diagrams with any particular token placed in brackets and thus facilitating control, experiment, and quick development of these signs. (Stjernfelt 2002: 341)

There is a consequence of this abstraction that is highly characteristic of the symbolic order, which is the power for various kinds of reflexivity. Among the things that can 
be described by a universal category is any and every aspect of language itself; language is in this sense self-referential. Now I am aiming at something quite different here from the normal notion of metalanguage, whereby we use language to discuss language, as when one says What does this word mean? or How do you say this in German? and so on. Equally I am not alluding to a circularity of the kind: "Semiosis explains itself by itself; this continual circularity is the normal condition of signification" (Eco 1976: 71). These relatively innocent phenomena are perhaps well understood and may fall within an unproblematic notion of unlimited semiosis, one that does not sow the seeds of its own destruction, so to speak.

I want to point out certain processes in diachrony that appear to lead inexorably to escalating levels of abstraction, the roots of which lie in the evolution of culture and which cannot develop without periodically undermining the basis of communication itself. Whether these point to some catastrophic fate for human beings and their language or to some millennial supersession of language itself (a leap into the post-human, or perhaps into telepathy, etc.), I cannot say. However, there is a logic at work that seems to me to be analogous to those financial processes that periodically wipe out large amounts of wealth on the stock market that suddenly turn out to have been nothing more than hot air. This analogy is a considered one, following Petrilli and Ponzio (2008), the idea being that linguistic products can be fetishized (like commodities) so as to efface the real human relations underlying them.

I begin the explanation of this by drawing attention to the fact that we nominalize certain discourse processes in an onomasiological way. That is, the progress of discourse creates new meanings that stand in need of naming, of a signifier. These nominals become more and more abstract in their nature, insofar as their function is to summarize those parts of prior discourse that one wishes to evoke without having to repeat them laboriously. The recursive mechanism in language that gives birth to such processes is found according to Thom (1983: 176) also in mathematical discovery. He alludes to "a direct extension of this mechanism of symbolic creation" whereby "the mathematician sometimes sees an expression, or a relation, turning up again and again with an embarrassing insistence," so that he will "introduce a new symbol to condense this expression into a single form and so continue the work on a new basis." The new basis here is a higher level of abstraction.

In the quoted passage Thom shows with great clarity how this recursion and advancement to new levels of abstraction occurs in relation to the semiosis of mathematics. The more general principle of abstraction is that a statement or set of statements is represented by a new sign or nominal expression. But then this new nominal itself enters into new expressions in its turn, thereby raising the level of abstraction. It is in these accretions of discourse that are nominalized and "profiled" (Visetti and Cadiot 2002) via abstract expressions that the full potential of the symbolic order, as well as its aporiae, are to be located. Just as Thom's new mathematical form has a group of preceding others as its meaning, just so in 
language more generally must we retrace our steps into prior discourse in order to find the original meanings of certain nominal expressions we use.

Thom says that creating a new symbol like this "promotes a kind of tearing away, with the establishing of a new semantic field which will be the support of the new actant and so free the mental movement from the obsessional presences which impede it" (1983: 276). Just so with language in general; a new symbol arises which allows the mind to fly free of the obsessional particulars of its previous moments. But what we have added to this imagery of semiosis without limit (in this case abstraction without limit) is the caution that this freedom requires that we be able to trace our way back whence we came and to show others the same path, lest we forget what we mean. The ability, the opportunity and the will to do this, however, cannot always be taken for granted. To explain this further I need to say something more about how levels of abstraction arise. But let us note first the natural and ethical aspects of this.

The natural aspect consists in the limits of mental capacities. Having performed the kinds of summative operations that give birth to the new symbol, one will tend to find a gradual atrophy in memory of some of the original processes underlying it, to the extent that these were even known by the individual in the first place. While this might not be the case in highly expert domains (such as the work of the professional mathematician) it may well be the rule in other domains of discourse.

The ethical aspect lies in the highly differential ways that an abstract term might be profiled in discourse according to the intentions of the communicator. It is arguably part of the definition of semiotics that it deals with the capacity to deceive (Petrilli and Ponzio 2001: 39-41). This is not confined to the symbolic realm, but is observable across many life forms. However with escalating abstraction in the symbolic realm the potential for deception, as well as other less intentional forms of miscommunication, is vastly enhanced. And in all cases there is a certain “answerability" involved (Ponzio 2008).

\section{Semiosisandabstraction}

Abstraction has been shown to occur in at least three degrees (Wood 2006, 2009):

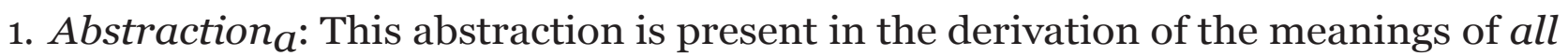
categories, even those that we associate with "concrete nouns." It is the process whereby categories of concrete objects or qualities are separated, by a process of idealization from the individual instantiations of the category. In cognition such categories can generally be simulated as image schemas (Lakoff 1987). As such they can also be demonstrated to a language learner through acts of ostensive definition: "that is a chair," "this is a table," etc. They also exhibit prototype effects, whereby one member of a category is a more or less central exemplar of the category than another, so that one does not generally teach someone the meaning of chair by pointing to a dentist's chair or the meaning of table by pointing to an operating table, etc. This is the most fundamental level of abstraction, so much so that in common parlance it is not thought of as abstraction at all. This is because 
of an unreflecting identification of a concrete object with the category. The object of reference is not abstract but the category is.

2. Abstractionb: Here we have to do with abstract nominals of the common kind, words such as charity, disappointment, etc. The abstraction here is the process whereby an idealized narrative schema comes to form the meaning for a lexical item. These might well be identical to the "situation models" postulated by van Dijk and Kintsch (1983). A model of a common situation becomes, diachronically, nominalized, so that the meaning of the new signifier (if indeed it is entirely new) is provided by an idealization of the narrative of the common situation.

Note that the abstract category that emerges in such a process does not correspond to any class of physical objects or their qualities and cannot be simulated or defined ostensively as in the case of abstraction ${ }_{\mathrm{a}}$. There is no concrete object that can instantiate a category such as charity or disappointment. Nevertheless something remarkably like ostensive definition is possible. One may help to create the narrative schema by telling a little story and then saying "that is charity" or "that is disappointment." The indexical is not used to indicate an object but the little story that has just been told. Thus categories at this degree of abstraction can be defined in a quasi-ostensive way (see Benzon and Hays 1990). But normally the matching of situation model with nominal will occur in a less explicit experiential way.

- Abstraction $c$ : This is hyper-abstraction. Hyper-abstract concepts cannot be demonstrated or defined through basic narratives (let alone through acts of ostention) without severe reduction of their meanings. Their meanings are constituted via bodies of heterogeneous discourse, the more heterogeneous the discourse that constitutes its meaning the more unstable the concept and the more likely that it will have different meanings for different interpreters. Here we might invoke Peirce and say that different interpretants may be active for different audiences, and we might also want to explore convergences between Visetti and Cadiot's (2002) notion of "profiling" and Peirce's notoriously elusive interpretants. It may just be that the former places more emphasis on the activity of the sender and the latter more emphasis on the activity of the receiver.

\section{The aporiae of hyper-abstraction}

A simple idealized narrative schema will not do for the meanings of the nominals that we are now concerned with. The problem is that the meanings of hyperabstract words are defined by the various contexts of discourse in which the subject has encountered them, and this will vary from one individual to another. And any indeterminacies in meaning can also be exploited by the sender in the way that the concept is profiled in the text, that is, the way in which the concept is used to afford or block access to certain themes and topics. A successful profiling therefore means the reduction of a complex of semiotic potential to just those meanings that are associated with particular themes. 
Consider a word like romantic for example. One might use this word without regard to the contexts in which one's interlocutor has encountered it before. But the understanding of the interlocutor might vary greatly according to whether he or she has been exposed to, say, the chivalric romance, Byron and Shelley, Beethoven and Chopin, the gothic novel, and so on, as opposed to such popular genres as Mills and Boon love stories, detective fiction and Hollywood "romantic comedies." Then there is the language of travel to exotic ("romantic") destinations, types of restaurants that offer a "romantic evening," etc. This dependence upon prior discourse is characteristic of hyper-abstraction. Unlike those abstractions of the second type, one cannot define romantic with any adequacy by means of a simple narrative schema.

Notice that the problem is not simply one of polysemy, which in fact characterizes much of the lexicon. To understand a hyper-abstract word like this one must know which of the prior discourses it is summarizing or "standing-in-place-of" and what such a discourse typically consists of, its various symbolisms, assertions, themes and topics. Therefore we continually make judgments about the competence and knowledge of our interlocutors if we care about their understanding when we use such words, and it is quite impossible to separate language from knowledge in such cases, since certain linguistic items are precisely dependent on knowledge of discourses for their meaning.

Once one has to do with hyper-abstract terms a radical indeterminacy in interpretation may arise, which cannot be remedied by recourse to any rules or approved procedures of interpretation. Thus any limitlessness of interpretation that we may be inclined to postulate may indeed turn out to be a "bad infinity"; bad in the precise sense that it represents the breakdown of common understanding and allows for a range of (mis-) understandings that are themselves potentially infinite in number, paradoxically creating thereby a kind of identity between meaning and meaninglessness in a single instance. Rather than the neat radial structure of prototype effects, or the "motifs" (Visetti and Cadiot 2002) that are easily stabilized in the profiling process, we have rather an uncertain meaning potential that is not necessarily stabilized according to context, but which remains unstable across the various readers/hearers, despite whatever profiling has been supplied by this context. The source of this is natural; it consists in the capacity constraints of long-term memory, as well as the impossibility, due to spatiotemporal constraints, of subjective exposure to all the possible discourses that are potentially able to furnish meanings of the word.

\section{The concrete imperative}

It is here that we should be concerned with what we might call the "semiotics of the deed." If there is always a certain answerability in human communication then it is in the case of hyper-abstraction that it may become a particularly serious problem. We may use hyper-abstraction inter alia to impress, to deceive or to enlighten and we are ethically obliged at least to consider which of these we are engaged in. The serene lack of interest on the part of the speech act theorist towards the perlocutionary effect of one's illocutionary act may in this light appear as a form of irresponsibility. Compare this with, for example, the concerns underlying McKenna and Graham's (2000) discussion of the obfuscatory use of abstract nominals in technocratic discourse. 
A macro-ideational structure that comes about intertextually (Wood 2006, 2009), as a merging of the content of various texts into a summative cognitive structure that can be permanently stored in semantic memory, is a concrete process at the level of the individual and an ethical semiotics would be one in which such concrete processes are considered. A lack of consideration for the experience of one's interlocutor perpetuates the key aporia in hyper-abstraction, where the concrete interpersonal nature of communication has been obscured by verbiage.

The price that human beings pay for their symbolic capacity becomes apparent the more highly developed their culture has become, in other words the more hyperabstract terms it has generated. If this were not so there might never be a serious hermeneutic problem. "Cultures differ in their capacity to order and generate abstract concepts," and "there are abstract ideas that cannot occur in the thinking of an Eskimo, or even a literate Florentine, or, for that matter, Darwin, Freud or Einstein" (Benzon and Hays 1990: 298).

\section{Adiachroniclogic}

This fate of subjectivity can only be conceived of as an "unlimited semiosis" if the latter is a bad infinity, that is, a semiosis that is indistinguishable from its opposite. This arises if we attempt to think of an infinity of interpretants, which is conceivable, but to what effect? If such an excessive plurality of meanings is accepted then this must surely be to admit the possibility of meaninglessness in any and every encounter. What if this very meaninglessness is what makes such words attractive to communicators whose purposes are not quite what they seem? This problem has long been there at the aporetic heart of the symbolic order - one thinks of a Socrates endlessly pondering over the meanings of abstract words such as justice, the good, and so on - and its appearance as a problem becomes more pronounced when subjectivity reaches certain crisis points in its maturity, for example due to increasing division of labor, or division between those who labor and those who do not. But it does not similarly arise as a crisis of subjectivity at lower levels of abstraction, which are based on substantially common experiences. Who cannot model the situations in which charity or disappointment are applicable?

Rather than limitlessness then, let us consider the nature of the limit. In doing so we should bear in mind that hyper-abstraction is a reality and we should not claim that it could somehow have been avoided by a decision or by wiser counsel. What is being claimed instead is that it represents a limit that is internal to the language that we use, a limit that we may learn to master. Hegel says:

A thing is what it is, only in and by reason of its limit. We cannot therefore regard the limit as only external to being which is then and there. It rather goes through and through the whole of such existence. The view of limit, as merely an external characteristic of being-there-and-then, arises from a confusion of quantitative with qualitative limit. Here we are speaking primarily of the qualitative limit. If, for 
example, we observe a piece of ground, three acres large, that circumstance is its quantitative limit. But, in addition, the ground is, it may be, a meadow, not a wood or a pond. This is its qualitative limit. Man, if he wishes to be actual, must bethere-and-then, and to this end he must set a limit to himself. People who are too fastidious towards the finite never reach actuality, but linger lost in abstraction, and their light dies away. (Hegel 1975 [1830]: §92)

To "be actual" in relation to semiosis means, for one thing, to accept the limit that I have been describing. To ignore actuality in this sphere may indeed amount to being "lost in abstraction."

Despite the quantitative limitlessness of semiosis, which is entirely plausible, albeit "tedious" to contemplate (Hegel 1975 [1830]: §94), we need to postulate an entirely qualitative internal limit to the symbolic order. This is provided for us by the notion of abstraction. Abstraction defines the limit condition for the symbolic order in this sense, that it makes the symbolic what it is (i.e., something more than the iconic and the indexical), yet it is also what makes the symbolic turn back to the concrete, i.e., to the iconic and indexical, in order to re-establish, or to stabilize, meaning. To put it more simply, the symbolic is apparently quite distinct from the types of signs that characterize the animal kingdom, but in actuality it always carries the indexical and the iconic within itself as part of its own limit, that which it cannot leave behind entirely.

"Unlimited semiosis" thus has this aporia within it: As soon as it has become unlimited, meaning will break down; and as soon as meaning has been restored, this implies that it must have become limited again. Applying a purely quantitative infinity here would mean a semiosis that is actually the opposite of itself, sheer meaninglessness. After all, our analysis of abstraction has already shown that the number of "interpretants" is potentially infinite and therefore unknowable to a subject.

So from a logical point of view the problem with the notion of unlimited semiosis is that it does not make clear whether it is a qualitative or quantitative limit that is at issue. If it is taken in the sense of a purely quantitative limitlessness, it is easy to see how this would exhaust the mind and defy interpretation, even though it is imaginable. Such an infinite opposes itself absolutely to the finite (as its other). But a qualitative limit must prohibit such a spurious infinity if meaning is to be preserved and semiosis is to be based on actuality.

In short, the concrete particularity of the iconic and the indexical must be found within the symbolic as its internal defining limit. In practice this is the concrete particularity of individual experience and memory, and the possibility of ethics in communication rests upon giving this concrete its due. 


\section{Conclusion}

One might characterize abstractions as summaries of stories. Hyper-abstractions would then be summaries of stories about discourses, where the original discourses may not be immediately available to provide a relative concretization for the understanding of the subject. To proceed to yet a higher level of abstraction than this could only be to leave the concrete entirely behind, no doubt a form of madness if it were possible.

I have suggested, borrowing from Hegelian logic, that unlimited semiosis, taken in its purely quantitative aspect, arrives at meaninglessness, the inability of a subject to determine the meaning of an expression either for him/herself or for another. This is not to say that this infinitude is not operative in practice; it is, and where it is so it is bad. Insofar as one naturally turns back from such an abyss in an attempt to restore meaning, this infinity is revealed to be spurious. Obviously I have not attempted to explore this problem in all of its dimensions; I have focused on the question of hyper-abstraction. Escalating levels of abstraction may reach a point at which the subject is no longer modeling any particular type of situation at all, and must bring the discourse back to more concrete particulars in order to resolve the indeterminacy in meaning. The escalation in level of abstraction arises from the summarizing function of abstract signs - a function that may be confined to human language only - whose successful use depends upon the links that can be established between the present discourse and the prior discourse that is being summarized or recalled by the hyper-abstract term.

No doubt we must postulate certain features of the evolved cognitive apparatus that allow the human subject to attain higher levels of abstraction, but also features that place a constraint upon this activity. Analogies can be found in other areas of language study, for example in sentence grammar, where the number of spatially distinct actants that can be organized around a verb tends to be limited to four, notwithstanding the prodigious syntactic complexity that this still allows for.

Once we understand the problem of hyper-abstraction better, we will surely have obtained similar important insights into the capacities and constraints of cognition. What I am hoping to have provided here is a critical insight concerning unlimited semiosis and the problem of conceiving of this in practice as an infinite series of interpretants. Instead what appears to be needed is a better understanding of the limit, so that signs are seen as always in a process of becoming, each an (always partial) approximation to its own scope of applicability as determined by variation in subjective experience and thresholds of passage from meaning to non-meaning, or from comprehension to noncomprehension. The notion of interpretant, while apparently admitting subjectivity, tends to be postulated without due regard to this limit. I suggest that this might be a natural limit, but one with profound ethical implications. 


\section{References}

Benzon, William L. \& David G. Hays. 1990. The evolution of cognition. Journal of Social and Biological Structures 13(4). 297-320.

Deely, John. 2004. Semiotics and Jakob von Uexküll's concept of umwelt. Sign Systems Studies 32(1/2). 11-34.

Eco, Umberto. 1976. A theory of semiotics. Bloomington: Indiana University Press.

Hegel, G. W. F. 1975 [1830]. Hegel's Logic, William Wallace (trans.). Oxford: Oxford University Press.

Lakoff, George. 1987. Women, fire, and dangerous things: What categories reveal about the mind. Chicago: Chicago University Press.

McKenna, Bernard. J. \& Philip Graham. 2000. Technocratic discourse: A primer. Journal of Technical Writing and Communication 30(3). 219-247.

Mills, Jon. 2004. Clarifications on trieb: Freud's theory of motivation reinstated. Psychoanalytic Psychology 21(4). 673-677.

Peirce, Charles S. 1894. What is a sign? MS 404. http://www.iupui.edu/ peirce/ep/ep2/ ep2book/cho2/ep2ch2.htm (accessed 26 June 2013).

Petrilli, Susan \& Augusto Ponzio. 2001. Thomas Sebeok and the signs of life. Duxford: Icon. Ponzio, Augusto. 2008. Communication with the other and moral answerability in Mikhail M. Bakhtin. Russian Journal of Communication 1(3). 292-307.

Sebeok, Thomas A. 1999. The sign science and the life science. Applied Semiotics / Sémiotique appliqué 3(6/7). 85-96.

Stjernfelt, F. 2002. Tractatus Hoffmeyerensis: Biosemiotics as expressed in twentytwo basic hypotheses. Sign System Studies 30(1). 337-345.

Thom, René. 1983. Mathematical models of morphogenesis. Chichester: Ellis Horwood.

Van Dijk, Teun A. \& Walter Kintsch. 1983. Strategies of discourse comprehension. New York: Academic Press.

Visetti, Yves-Marie \& Pierre Cadiot. 2002. Instability and theory of semantic forms: Starting from the case of prepositions. In Susanne Feigenbaum \& Dennis Kurzon (eds.), Prepositions in their syntactic, semantic, and pragmatic context, 9-39. Amsterdam: Benjamins.

Wildgen, Wolfgang. 1982. Catastrophe theoretic semantics. Amsterdam: Benjamins.

Wood, Tahir. 2006. Adherence relations in literary and non-literary discourse. Journal of Literary Semantics 35. 165-180.

Wood, Tahir. 2009. Adherence and abstraction in discourse processes. Journal of Pragmatics 41. 484-496. 


\section{Bionote}

Tahir Wood (b. 1953) is an associate professor and director of the academic planning unit at the University of the Western Cape $\square$ twood@uwc.ac.za $\square$. His research interests include semiotics, semantics, pragmatics, and discourse. His publications include "Between signification and the referentoial act: the proposition as pure ideation" (2010); "Beyond signification: the co-evolution of subject and semiosis" (2011); "Hermeneutic pragmatics and the pitfalls of the normative imagination" (2011); and "Author's characters and the character of the author: the typical in fiction." 